\title{
Traditional Chinese Medicine Explanatory Models of Depressive Disorders: A Qualitative Study
}

\author{
Wan $\mathrm{Kam}^{1}$ (D) Zhang-Jin Zhang ${ }^{2}$. \\ Sofie Bäärnhielm ${ }^{1,3}$ (D)
}

Published online: 8 April 2019

(C) The Author(s) 2019

\begin{abstract}
Traditional Chinese medicine (TCM) is an alternative medical system utilised by many Chinese. However, the knowledge of TCM concepts of depression is limited amongst clinicians with training in Western biomedicine. The purpose of this study was to obtain a better understanding of the conceptualisation of depression from a group of TCM practitioners. Semi-structured interviews in Chinese were carried out with 10 TCM practitioners in Hong Kong. A case description of major depression disorder (MDD) was used as a basis. Interview texts were transcribed, translated and analysed using qualitative content analysis. Most informants identified the case as a depression pattern, a term that lacked clear definition and standardised criteria. The mechanism of disease for MDD symptoms were regarded to be liver-qi dysregulation and an imbalance of yin and yang. The TCM practitioners implemented individualised diagnosis, treatment, and a holistic concept without clear distinction between the mind and the body. This contrasted with the biomedical tradition of separating psychologisation and somatisation. The meanings
\end{abstract}

Electronic supplementary material The online version of this article (https://doi.org/10.1007/s11013019-09628-7) contains supplementary material, which is available to authorized users.

Sofie Bäärnhielm

sofie.baarnhielm@sll.se; sofie.baarnhielm@ki.se

Wan Kam

w.kam.mindhealth@gmail.com

Zhang-Jin Zhang

zhangzj@hku.hk

1 Transcultural Centre, North Stockholm Psychiatric Clinic, Stockholm, Sweden

2 The University of Hong Kong School of Chinese Medicine, 10 Sassoon Road, Pokfulam, Hong Kong, China

3 Department of Clinical Neuroscience, Karolinska Institutet, Stockholm, Sweden 
given to the concept of depression did not correspond with current DSM or ICD definitions, and the TCM normativity can result in variations in explanatory models.

Keywords Traditional Chinese Medicine - Depression - Cultural psychiatry · Explanatory model

\section{Background}

For depression, about $90 \%$ of all patients globally are of non-Caucasian origin (Lee, Kleinman, and Kleinman 2007). Cross-cultural psychiatry has been an important subject for research since the term was coined by Arthur Kleinman in the late 1970s (Kleinman 1977), and has evolved to become the concept of cultural psychiatry today as recognition of the broad nuances of illness presentation, patient experiences and medical interpretations world-wide. China has the largest population per country in the world, and how the Chinese express depression is one of the fields being regularly explored, from Kleinman's classic study on neurasthenia and depression (Kleinman 1982) to a more recent review on how Chinese in Western countries experienced depression (Ahmad et al. 2018). Western biomedicine has been well-established across China but 10-20\% of the Chinese population still seek Traditional Chinese Medicine (TCM) service, a tradition that dates back centuries (Chung et al. 2014; McQuade et al. 2012). With the modern globalisation and population movements across borders, improving Western medical professionals' understanding of how TCM practitioners conceptualise and treat symptoms associated with depression can be of major public health significance.

There are studies demonstrating the effectiveness of TCM treatment methods on mental health, for example for depression, chronic pain, and climacteric syndrome (Eisenhardt and Fleckenstein 2016; McKee et al. 2013; Tao et al. 2016; Yeung et al. 2014). However, few studies have examined how TCM practitioners diagnose depression and interpret it as a concept. In a broader spectrum, what do TCM practitioners utilise as explanatory models (EM)? EM refer to how a person perceives, interprets, expresses, explains symptoms and experiences, while they also influence the treatment process (Kleinman 1978). Research published in Chinese is difficult for non-Chinese clinicians to access. Descriptions of TCM theories and definitions are found in various TCM textbooks, most of which are written in Chinese by small groups of senior practitioners basing their work on ancient scripts and individual experiences. All of this makes it difficult for psychiatric professionals in Western countries to gain insight into TCM. For a better understanding, learning about the EM of TCM practitioners is important.

\section{Purpose of the Study}

The overarching aim of the study was to gain an improved understanding of the conceptualisation of depression among a group of TCM practitioners. The specific aims were to find out more about the following: a) how a group of TCM 
practitioners perceive depression as a phenomenon and a term; b) what treatments they would recommend for a depressed person; c) how they compare TCM and Western biomedicine theories concerning the diagnosis and treatment of depression.

\section{Methods}

\section{Study Design}

\section{Choice of Method}

We chose to carry out a qualitative study based on open-ended, semi-structured interviews in Hong Kong and used a case vignette approach. Vignette-based methodologies are often used to examine judgements, 'real life', behaviour, and decision-making processes, including clinical judgements made by health professionals (Evans et al. 2015). Content analysis (Krippendorff 2004) was performed on the collected data. Hong Kong was chosen as the research location because of its well-established research environment, good public access to medical care, and its strong Chinese traditions. In Hong Kong, 50-60\% of people would visit TCM clinics for medical consultation (Wong et al. 2011).

\section{Recruitment of Informants}

Subject recruitment was carried out from August to October 2016 at two locations: (1) Clinical Centre of Teaching and Research (Central), School of Chinese Medicine, The University of Hong Kong; (2) The Hong Kong Buddhist Association - The University of Hong Kong Clinical Centre for Teaching and Research in Chinese Medicine. The locations were chosen because they were the two clinics attached to the School of Chinese Medicine, the University of Hong Kong, which has its ruling ethical committee clearly appointed. Both centres regularly receive TCM students and young TCM practitioners for training. The clinical centre at Central has fewer but more senior practitioners whilst the Buddhist Association clinical centre has a larger proportion of younger practitioners. Recruitment was carried out at both centre iteratively to optimise diversity of informants.

A purposeful sampling was chosen as this an approach for identifying and selecting information-rich informants and for an effective use of limited resources. It entails identifying and selecting individuals particularly informed about or experienced with a phenomenon of interest (Palinkas et al. 2015). In this study this was conducted by the first author initially approaching random practitioners at the clinics for recruitment. As the process continued, data analysis (familiarising with the data by listening to the recordings and starting the transcription) started parallel to the interview process to identify informant categories and content themes. Both clinics had a list of the names and education qualifications of its practitioners. Each informant was asked to fill out a background data sheet. This allowed a strategic selection of informants with the most diverse backgrounds possible in terms of age, gender, religion, and education. 
Informed consent was obtained from all individual participants included in the study, in oral and written form.

\section{Sample}

\section{Sample Characteristics}

The collected background data sheets showed the following:

\begin{tabular}{llrll}
\hline $\begin{array}{l}\text { Informant } \\
\text { gender }\end{array}$ & $\begin{array}{l}\text { Number of } \\
\text { informants }\end{array}$ & $\begin{array}{l}\text { Average age } \\
(\text { min-max })\end{array}$ & $\begin{array}{l}\text { Educational background } \\
\text { Mainland China: Hong Kong }\end{array}$ & $\begin{array}{l}\text { Recruitment site central: } \\
\text { Buddhist Hospital }\end{array}$ \\
\hline Male & 5 & $43(26-64)$ & $5: 0$ & $3: 2$ \\
Female & 5 & $41.2(30-60)$ & $3: 2$ & $3: 2$ \\
\hline
\end{tabular}

Of the 10 TCM practitioners interviewed, 5 reported that they did not have any religion, 2 reported believing in Christianity, 2 in Buddhism, 1 gave their religion as Buddhism/Taoism.

\section{Data Collection}

A case description (Online Appendix 1) aiming to demonstrate an example of major depression disorder was validated by a psychiatrist colleague in Stockholm and one in Hong Kong. It was shown to each informant at the beginning of the interview.

Interviews were carried out by the first author in Cantonese or Mandarin depending on the preferred language of the informant. The TCM practitioners were asked about their views on the case presented, the diagnosis and treatment they would give, whether TCM had a term for depression, and their opinions on how TCM and Western biomedicine compared on the subject. The interviews were audio-recorded. Complementary side-notes were made during individual interviews.

\section{Data Analysis}

Interviews were transcribed in Chinese and translated into English verbatim. For the quality control and validation of transcribed interview texts, a third-party translation firm was contracted. Five of the ten transcriptions were randomly chosen for proofreading and validation. As all transcription and translation were done by the first author, the validation of the five transcripts was extrapolated to the rest.

The texts were coded inductively after each interview had been transcribed. The coding was conducted by the first author on the translated English transcript with constant reference to the original text in Chinese in order to cross-check expressions and context. The codes, categories, and themes were continuously discussed and consensually adjusted in discussions with first the last author and finally with all authors. 


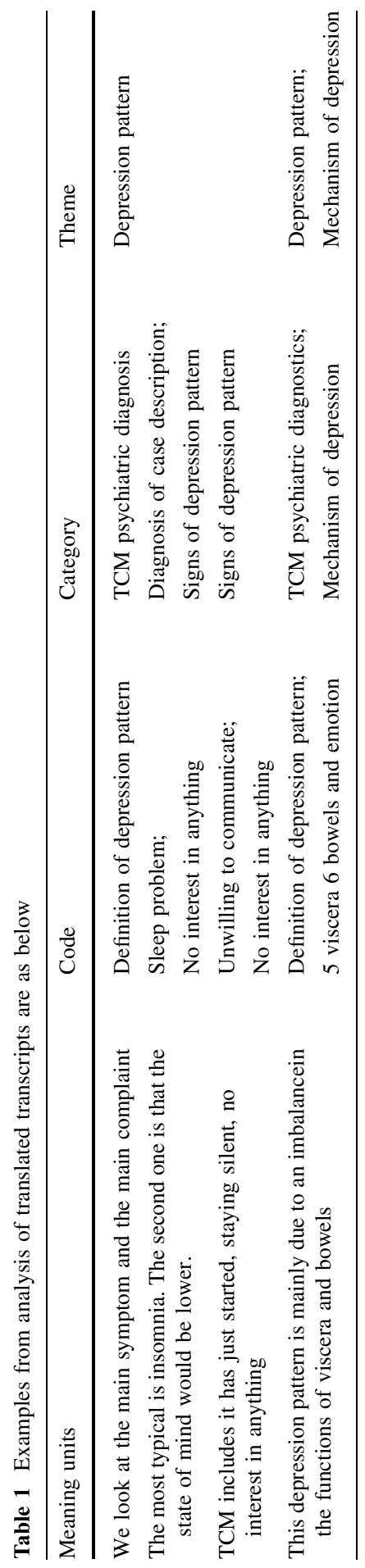


The coding process was carried out with the aid of Open Code. Open Code is a software for coding qualitative data from text information, developed by the University of Umea in Sweden. Open Code only supports code synthesis in two stages. Subsequent synthesis analysis was performed manually. General principles of content analysis (Krippendorff 2004) were used throughout the analysis process. This applied that (1) codes were assigned to meaning units identified from the texts; (2) codes were grouped into preliminary categories; (3) broader themes synthesizing the findings were identified and redefined. An illustration of this process is given in Table 1. The meaning unites were phrases from transcripts. Codes were indexing units, which were then organised into descriptive categories. Themes were built from categories, and used to construct the final interpretations. To facilitate the understanding of the contents and the analysis process, the WHO document for international standard terminologies on traditional medicine in the western Pacific region (WHO Regional Office for the Western Pacific 2007) was used as a translation guide and a key for looking up definitions of TCM terms.

\section{Findings}

The following four themes were identified from the interviews: depression pattern, diagnostics and treatment; mechanism of depression, and the significance of the liver; the significance of qi; and a holistic view.

None of the informants mentioned anything related to religion in their process of setting diagnosis and reason around treatment. Purposive recruitment to include education background from mainland China and Hong Kong was carried out. Both practitioners educated in mainland China and Hong Kong cited a proportion of 30$40 \%$ of their university curriculum being in Western biomedicine.

\section{Depression Pattern, Diagnostics and Treatment}

All the informants thought the case description showed a condition very commonly seen in clinical practice. Eight of the ten informants identified the case as depression or depression pattern. Amongst the eight, six informants recognised depression pattern as the sole diagnosis, one offered a differential diagnosis of menopausal syndrome, one first set headache as the diagnosis and reached the conclusion of depression pattern later during the interview. Of the remaining two informants, one stated that a diagnosis could not be reached due to lack of information, and one set menopause syndrome as diagnosis.

According to the informants, common signs of depression patterns were: low mood, lack of essence-spirit, lack of energy, insomnia, lack of appetite, concentration difficulties, no interest in anything, pessimistic thoughts, worries a lot, unwilling to communicate with others, unable to work. General discomfort and pain were also identified as depression symptoms. In terms of TCM examination methods, the pulse could be string-like, but there might not be any obvious pathologic findings in terms of physical examinations. 
Table 2 Definition of the four examinations in TCM (WHO Regional Office for the Western Pacific 2007: 80-101)

\begin{tabular}{ll}
\hline Terminology (Chinese character; Mandarin phonetic) & Meaning \\
\hline Inspection (望; wang4) & $\begin{array}{c}\text { Observing the patient's emotions, facial } \\
\text { expression, complexion, physical condition, } \\
\text { condition of the tongue, secretions and the } \\
\text { distribution of superficial venules } \\
\text { Listening to the patient's speech, voice, } \\
\text { articulation, breathing, cough; smelling the } \\
\text { patient's odour from the mouth, and body } \\
\text { Asking the patient about the complaint and the } \\
\text { history of the illness } \\
\text { Touching and pressing the body surface with the } \\
\text { hand or fmgers, examination of the pulse and } \\
\text { locations of complaint }\end{array}$ \\
Palpation (切; qie4) &
\end{tabular}

However, the informants considered depression pattern to be a broader concept than major depression disorder. There were no set criteria to be met: no strict requirement of duration or number of symptoms. There could also be symptoms not mentioned in the DSM-5. One informant described it as follows, "...the beginning, the initial pre-depression symptoms, we also call it depression... young people who lose their tempers [and] scold people, that we also call depression pattern" (TCM practitioner 10). According to the interviews, depression pattern could be caused by underlying somatic illness, even temporary physical discomfort.

Eight informants emphasised that "the correlation of all four examinations (四診 合參)" was fundamental for diagnostics. For four informants, the case description only contained 'inquiry', and a precise pattern identification was not possible due to the lack of results from the other three examinations. Three informants emphasised the importance of inquiring about the menstruation status when the patient is a woman. Menopausal syndrome was named as an important cause of symptoms/ differential diagnosis by four interviewees.

Concerning treatment, seven informants considered the case description insufficient for treatment planning. This was due to it only provided information for inquiry, and lacked results for the other aspects of the four examinations (Table 2). Eight informants suggested a combination of medicinal formulas, acupuncture, psychotherapy, massage, music therapy, exercise, diet adjustment, or adjustments in the living environment. The informants considered it normal that each TCM practitioner might identify different patterns and choose different treatment methods given the same case, depending on the practitioner's experience.

"However, even though the opinions are different maybe all would solve her problem too. So, that is our TCM's, a little special feature [TCM] has. That is the person's, the thoughts the ways of thinking, are different, so, the formulas prescribed are also different ... Because our opinion is a thinking more of philosophical character. Our medical science is one that is more of 


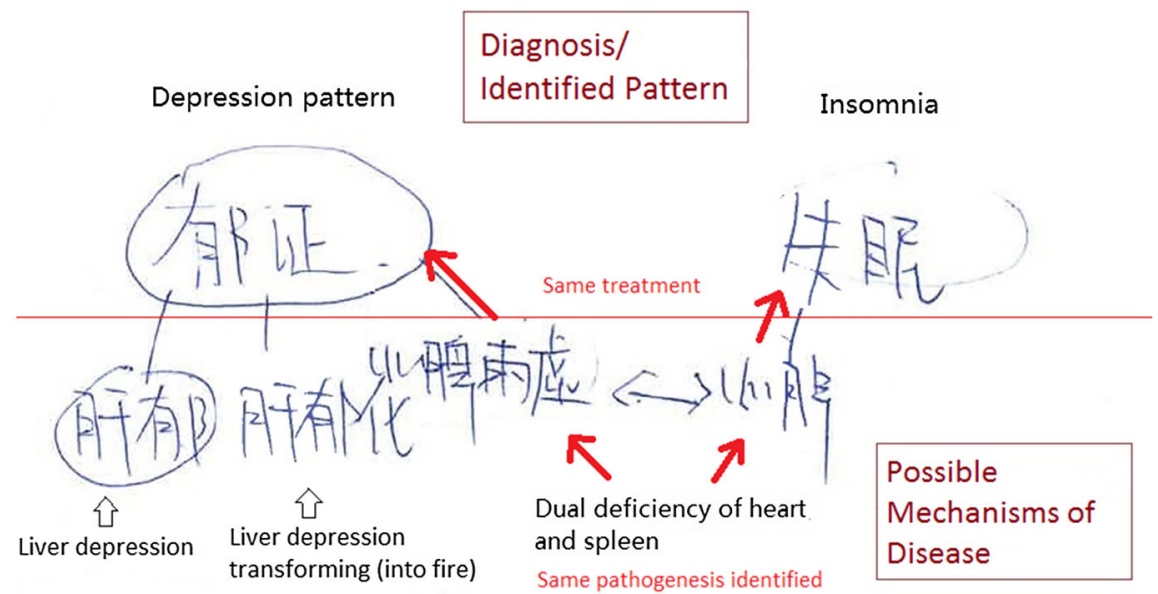

Fig. 1 The relation between diagnosis and mechanism of disease as illustrated by one of the informants

philosophical character. So it's like, all roads would lead to Rome as such.” (TCM practitioner 9)

... they all follow the same theories and principles of TCM. It is just that, they are all just measures. Just like when you have a battle to fight, say for example knife, spear, sword, halberd, axe, tomahawk, hook, and prong, these things, it all depend on which one suits you better. Ah. In order to achieve the goal, which one of them you can use more unrestrainedly. (TCM practitioner 1)

\section{Mechanism of Depression, the Significance of the Liver}

According to three informants, the key to TCM judgement and treatment was the mechanism of the disease (病機, pathogenesis), not the diagnosis. One informant said, "[we are] very meticulous about whether the capture of mechanism of disease is accurate" (TCM practitioner 9). Regardless of diagnosis, if the mechanism of disease was identified to be the same then the treatment for the condition would also be the same. One of the informants drew a diagram to demonstrate the relation between diagnosis and mechanism of disease (Fig. 1).

According to seven informants, there were five viscera in the human body, closely linked to each other and each with different functions. The liver was considered to govern emotions, and the free-coursing of qi and blood. When the liver functions weakened, the flow of qi and blood became disturbed and obstructed. Symptoms of liver depression, such as the ones in the case description, would then occur. Four informants thought that liver depression was more commonly seen in middle-aged women. If liver depression became more advanced, it could transform into liver fire, leading to symptoms such as agitation, dryness in the mouth, headache etc.

Disturbance in one viscus would lead to dysfunction in other viscera. Six informants answered that inquiry into accompanying symptoms was important. 
Depending on which other viscera were affected, different accompanying symptoms might appear. The spleen was the viscus most closely related to the liver.

there is a quote in TCM, it says, 'see an illness of the liver, you know that the liver passes over to the spleen, so you should first strengthen the spleen'. When the liver has an illness you need to consider the spleen. The five viscera are interlinked (TCM practitioner 4)

Four of the informants deemed the case description to be a case of liver depression invading the spleen.

\section{The Significance of qi}

The concept of qi appeared in almost all aspects in the interviews, including examination, pattern identification, mechanism of disease, and principles of treatment. In TCM, qi is the basic element formed everything. It flows within the body, maintaining all functional activities of an organic body (WHO Regional Office for the Western Pacific 2007: 13-18). According to the informants who mentioned disturbances in qi as a disease mechanism for the case described, symptoms of qi depression and qi deficiency included fatigue, difficult to concentrate, discomfort and pain in the chest, short of breath, constipation, dry mouth, and agitation. Changes in qi movement would affect vital functions in the body and cause changes of mood.

In pattern identification, depression pattern could be further categorised into 6 subtypes, one of which was qi depression (氣榣). As for the mechanism of disease, qi deficiency was identified by six informants. The main symptom of qi deficiency was lack of energy. It might occur either in general or in specific organs such as the kidney, and could be caused by various factors including aging, diet, sleep habits, stress etc. The following quote illustrates:

"Internal causes are herself per se is entering that age stage, qi and blood start to become not enough fully exuberant, when there is insufficiency in liver-kidney, [it becomes] easy to be induced by receiving some external causes. We call it healthy qi insufficiency, then pathogenic qi easy-, easy to invade liver." (TCM practitioner 9)

The obstruction of qi was also mentioned as the main cause of symptoms in liver depression. Since a disturbance in qi was considered a main cause of depression, different treatment methods would be used with the goal of removing qi obstruction and nursing qi.

\section{A Holistic View}

All informants considered depression symptoms to be caused by a combination of intrinsic and extrinsic factors. Six emphasised that TCM always considered from a holistic perspective. One informant explained, "Because a person is living in this world, everything you come in contact with would affect your body." (TCM practitioner 1) This was considered a major difference between Western biomedicine and TCM. 
Intrinsic factors include aging, hormonal influences such as menopausal changes, physical illness, and the personality of the individual. Extrinsic factors include climate, diet, stress, living environment, living habits, interpersonal relations, and more.

Pressure from family and pressure from work were considered two major extrinsic causes of depression pattern. Two informants thought that depression symptoms were especially common in large cities. Economic demands would be higher in cities. Limited space meant less distance between persons, which would in turn increase anxiety and the risk of conflict.

One distinct feature was the theory of yin and yang. Originating in ancient Chinese philosophy, yin and yang were two opposite but interrelated forces found in everything in nature. Yin was the feminine, passive counterpart of the masculine, active yang (WHO Regional Office for the Western Pacific 2007: 21). Three informants thought that modern people were more susceptible to mood disorder because they no longer follow the natural diurnal rhythm. Reversing the diurnal rhythm would violate the harmony of "nature and human becoming one (天人合 一)". This means that the person cannot recuperate their yin and yang in an optimal way, thus becoming more susceptible to illness. One informant cited that "heavy yang leads to manic psychosis, heavy yin leads to depression" (TCM practitioner 5). Depression pattern could be due to a disturbance of yin or yang, though more often it correlated to a disturbance of yin.

The holistic view did not only apply to diagnostics but also treatment: "We have [the theory of] choosing the appropriate solution on the basis of person on the basis of time on the basis of place" (TCM practitioner 9). As the environment for the individual changed, and treatments started to affect the body, the mechanism of disease would also change. This constantly changing process was referred to as the concept of transmutation (變).

The holistic view and transmutation were the reasons why informants thought TCM could not and should not be studied and analysed in a statistically evidencebased way. They considered Western biomedicine and TCM to be two totally different medical systems that could collaborate but could not be combined. One informant said,

the advantages of TCM, I think it looks from a macroscopic perspective. It looks from an integrated/comprehensive perspective, yes, from a perspective that sees a person as a real living organism and putting him in a natural environment. Whereas, Western medicine, it usually, looks at a person from a more, relatively a more mechanic perspective, ah. It also considers the biological perspective. However, it overweighs on a microscopic, mechanic, ah, this kind of perspective (TCM practitioner 1)

From another informant:

in WM they view from the anatomical perspective... But TCM is reflected from a dynamic. It is (short pause) that is there is a live person giving you a reaction to let you to, to infer her inside internal change and to treat based on that (TCM practitioner 5) 
Nevertheless, the informants considered biomedicine to be important in their practice. Three informants mentioned that they would recommend the patient in the case description to first go through a biomedical health check to screen for abnormalities. One practitioner also explained:

to enable the patient to trust you as a doctor you must have WM knowledge, and TCM's this pure treatment method. WM's [knowledge you] must have, need to tell her, this illness in WM science is what illness, and how we would treat in TCM. But TCM during treatment, do not be like WM and treat against symptom, ah headache then painkiller, ah, for example stomach is not good prescribe stomach medicine, this is not okay. (TCM practitioner 8)

\section{Discussion}

This study gave insight into the thought processes applied by these interviewed TCM practitioners when encountering a case of major depression disorder. It revealed some of the complexity of TCM in clinical applications.

\section{On Methods}

A limitation with the study was that all informants were recruited from two university medical centres, thus there might be a selection bias for TCM practitioners who were more knowledgeable than average about mental illness in Western biomedicine. All the practitioners interviewed had studied Western biomedicine as part of their university TCM programme. This implied a spread of biomedical thinking to the current generations of TCM practitioners, which could have modified the explanatory models by the informants. In the interviews, the conventional term 'Western medicine' (西醫) was used by both the first author and the informants without further discussion on the definition of the term.

In data analysis, one difficulty was the translation accuracy of the interview text. The Chinese language has a higher context level than English. The same word in Chinese can have various meanings in English. One example would be the word ‘jīng-shén' (精神) in Chinese: it can be translated either as essence-spirit (TCM terminology), spirit (common language), or psychiatric (biomedical terminology). Whilst the first two may not differ much in their meanings, the last context is significantly different. Words with the same pronunciation can also cause confusion in the transcription process. One such example was the word 'zhèng'. It can be the character 症, meaning symptom; or 證, meaning pattern.

Another limitation was that the case vignette design only provided information for inquiry, one of the four TCM examination methods, to assess a patient. Nonetheless, inquiry is a major TCM diagnostic approach through which most clinical information can be obtained. This has been reflected in the Consensus-based recommendations for case report in Chinese medicine (CARC) (Fu et al. 2016) and ancient TCM bibliographies in which there have been numerous case reports recorded. Clinicians in Hong Kong are familiar with using incomplete four 
examinations information to deduce a diagnosis because case vignettes are used as an important component in the Hong Kong registered Chinese medicine practitioner examination. The findings obtained in this study are still meaningful in comparing TCM diagnostic modes with that of Western biomedicine, and contribute to important information about TCM understandings, practice and reasoning concerning medical decision making.

Regarding strengths, firstly, TCM practitioners of various ages and educational backgrounds were recruited. This gave a broad informant diversity. Secondly, all interviews were conducted by the first author, thus allowing flexibility during and after each interview to explore the topics brought up by informants. Thirdly, all transcriptions were carried out by the first author, and uncertain phrases were transcribed and translated after consulting the second author. This minimised the uncertainties in context and word choice. An additional strength was that the three authors come from different backgrounds and could review the data from standpoints bridging TCM and Western biomedicine. The first author is from Hong Kong and has been medically trained in Sweden. The second author is a TCM professor and practitioner, who has worked in mainland China and Hong Kong. The third author is from Sweden and a psychiatrist educated in Sweden. By going through the translations, coding, and analysis results together, understanding and knowledge from both Western biomedicine and TCM perspective were applied in the process.

\section{On Findings}

One of the most significant findings was that each informant gave related yet different explanations of the causes of symptoms, and several different patterns were identified based on the same case description. According to the informants, there was a general acceptance of judging the same symptoms differently, and the diagnostic criteria of major depression disorder in ICD and DSM were not to be equated with the diagnostic parameters of TCM depression pattern. There were no standardised criteria for depression pattern among the interviewed TCM practitioners. This also implied that designing a study to evaluate the effectiveness of TCM treatments would be difficult. This limitation was also recognised in a review on TCM treatment for depression (Yeung et al. 2015).

Table 3 The five viscera and their functions according to TCM (Zhou 2006)

\begin{tabular}{|c|c|c|}
\hline Viscus & Function & $\begin{array}{l}\text { Governs the } \\
\text { following emotion }\end{array}$ \\
\hline Heart & Controls blood circulation end mental activities & Joy, Fright \\
\hline Lever & Stores, blood, facilitates the coursing of qi, regulate menstruation & Anger \\
\hline Spleen & $\begin{array}{l}\text { Transport and digestion of nutrition, keep the blood flowing within } \\
\text { the vessels, and is closely related to the limbs and flesh }\end{array}$ & Thinking \\
\hline Lung & Control respiration, dominate qi, related to the skin and hair & Anxiety, Sorrow \\
\hline Kidney & $\begin{array}{l}\text { Store vital essence and qi, promote growth mental development, } \\
\text { reproduction, and urinary function; also have a direct effect on the } \\
\text { condition of the bone and marrow }\end{array}$ & Fear \\
\hline
\end{tabular}


Pattern identification (辯證) was central in TCM diagnostics for the study participants. The Chinese word 'pattern' (證) is defined by WHO as:

The diagnostic conclusion of the pathological changes at a certain stage of a disease, including the location, cause, and nature of the disease as well as the trend of development; conditions suggesting appropriate treatment; and condition specific to the individual (WHO Regional Office for the Western Pacific 2007, 79).

This is broader than the definition of 'disease' or 'syndrome' in Western biomedicine, and reflects a holistic perspective of diagnostic thinking. The emphasis on 'a certain stage of a disease' entwines with the concept of transmutation that the pathogenesis is in constant change as reactions to the environment and interaction between organic systems.

The TCM theoretical model of five viscera (Table 3), in combination with qi, and yin-yang, make TCM explanatory models difficult for doctors trained in Western biomedicine to understand. Based on the interviews, the liver was seen as the main viscus involved in the mechanism of depression. This EM is in line with the description of TCM in a 2013 article on Korean Hwa-byung and depression (Suh 2013) but different from another conceptualisation with the heart as the main viscus involved (Miller 2006). The liver in TCM differs from the liver in WM, with emphasis on an abstract system in constant interaction with other viscera and elements, not on the biological organ. In depression patterns, the roles of liver and qi are intertwined, covering the majority of possible mechanisms of disease.

A different conceptualisation of personhood may be a reason behind the contrasting holistic EM described by the TCM informants, and the historical tradition of separating somatisation and psychologisation in Western biomedicine. In a 2012 review, selfhood was defined as being in the mind (Ryder et al. 2012). North Atlantic societies have developed an egocentric view (Eriksen 2017: 20-23), the 'person' is equalled to the 'self', and 'self' is identified with self-knowledge and consciousness. The Western biomedical explanatory models of depression are historically rooted in psychologisation. Somatisation reflects a defence mechanism that limits the mentalising of emotions and important psychological experiences, and has been viewed by some as an immature response to problems (Ryder and Chentsova-Dutton 2012).

In China, the individual identity is defined by his position in relation to institutions, and his (last) name that signifies an ancestral lineage (Mauss 1985). This relational view can explain why the two major extrinsic causes emerged to be pressure from family and pressure from work, and that multiple informants lifted changing the living environment as part of treatment. Informants emphasised selfhood as a dynamic integration of the body, mind and surroundings of the individual, with no habitual emphasis on any of the three. Fundamental TCM theories on how viscera and bowels interact with mood, with special focus on the liver, imply a thought pattern that revolves around somatic features during constructions of EM. Qi, another unique concept in TCM, is also closely linked to the physical conditions of the individual. This means that both TCM practitioners 
and laypersons seeking consultation may focus on somatic expression of a depression pattern, without actual presence of somatisation.

Even though it takes into account interactions between the intrinsic and extrinsic factors, Western cognitive models seek to find a focal deviation (Arieli and Sagiv 2018), e.g. research on the serotonin system to explain the alteration on the patient's mental status. The interviews showed that TCM aims to find and regulate a balance amongst the different viscera systems, between yin and yang, the patient's interchange with his environment. TCM seems not to place a focus on singling out a particular biochemical or organ system. This echoes with the holistic contextualisation in anthropology stating that every phenomenon must be understood in a dynamic relationship with other phenomena.

\section{Implications of Findings}

The findings from this study with interviews with ten TCM doctors cannot be generalised. Nevertheless, the interviews yielded an insight into a Chinese ontology much different from that of Western biomedicine, and can form a pilot ground for further investigations. The following can be considered: (1) an interview study to explore how TCM practitioners view the concepts of psychologisation and somatisation; (2) since liver depression seems to be the most commonly identified pattern in clinical depression (Yeung et al. 2015), follow-up interview studies can be planned, with focus on this pattern type to further investigate how TCM practitioners would identify the pattern and recommend treatment.

The TCM holistic thinking resonates with the embodied enactive model in that organism-environment interactions shape the process of psychopathology, and that bodily experiences are important constructs of explanatory models (Kirmayer and Ramstead 2017). Western biomedicine professionals may risk misinterpreting a Chinese patient's illness history or symptom narration if comprehension of the TCM explanatory model is lacking, not the least as TCM rhetoric is commonly integrated into Chinese laypersons' rationale for self-care (Chung et al. 2014). As population growth and globalisation continue, Western clinics will likely encounter Chinese patients more frequently. A broader knowledge in alternative medical thinking amongst Western biomedicine doctors can also provide an additional service and treatment route for their patients regardless of ethnicity. The findings of this study are the beginning for such capacity building for Western biomedicine doctors, to increase their understanding for TCM so that communication and collaboration between the two medical systems may be strengthened and the public health service improved.

\section{Conclusion}

The interviewed TCM practitioners emphasised an individualised diagnostic and treatment system based on each patient, with consideration of the environment the patient inhabits. The TCM practitioners focused on finding the mechanism of disease in terms of viscera, qi, and a balance of yin-yang. For the informants, the 
meanings given to the concept of depression did not correspond with current DSM or ICD definitions.

The TCM normativity emerged from the interviews contrast with that of Western biomedicine. Psychologisation and somatisation are in a way culture-bound styles of narration. Further investigation into the psychopathology models of TCM can broaden the definition of normalcy in Western biomedicine. This in turn can nourish communication and collaboration between Western biomedicine and TCM, improving public health service and enabling better care for patients.

Acknowledgements Our thanks for travel funding to Cullberg Scholarship granted to WC by the Natur \& Kultur foundation, Sweden. For validation of the case description to Dr M Zeitelhofer Adzemovic, specialist in psychiatry, Psychiatry Northwest Stockholm, Sweden; and Dr DH Lam, specialist in psychiatry, Chinese University of Hong Kong. The validation of the translations of 5 transcripts was carried out by a licensed translator at Adams\&Co, Hong Kong.

\section{Compliance with Ethical Standards}

Conflict of interest On behalf of all authors, the corresponding author states that there is no conflict of interest. The authors alone are responsible for the content and writing of the paper.

Ethical Approval All procedures performed in this study involving human participants were in accordance with the ethical standards of the institutional and/or national research committee and with the 1964 Helsinki declaration and its later amendments or comparable ethical standards. Ethics approval was obtained from the ethics committee: Institutional Review Board of the University of Hong Kong/Hospital Authority Hong Kong West Cluster. The assigned IRB reference number was UW 16-184.

Informed Consent Informed consent was obtained from all individual participants included in the study.

Open Access This article is distributed under the terms of the Creative Commons Attribution 4.0 International License (http://creativecommons.org/licenses/by/4.0/), which permits unrestricted use, distribution, and reproduction in any medium, provided you give appropriate credit to the original author (s) and the source, provide a link to the Creative Commons license, and indicate if changes were made.

\section{References}

Ahmad, Farah Alan, Catherine Maule, Jamie Wang, and Wai Lun Fung

2018 Symptoms and Experience of Depression Among Chinese Communities in the West: A Scoping Review. Harvard Review of Psychiatry 26(6):340-351. https://doi.org/10.1097/HRP.00000 00000000202.

Arieli, Sharon, and Lilach Sagiv

2018 Culture and Problem-Solving: Congruency Between the Cultural Mindset of Individualism Versus Collectivism and Problem Type. Journal of Experimental Psychology: General 147 (6):789-814.

Chung, Vincent C. H., Polly H. X. Ma, Chun Hong Lau, Samuel Y. S. Wong, Eng Kiong Yeoh, and Sian M. Griffiths

2014 Views on Traditional Chinese Medicine amongst Chinese Population: A Systematic Review of Qualitative and Quantitative Studies. Health Expectations 17(5):622-636. https://doi.org/ 10.1111/j.1369-7625.2012.00794.x.

Eisenhardt, Sarah, and Johannes Fleckenstein

2016 Traditional Chinese Medicine Valuably Augments Therapeutic Options in the Treatment of 
Climacteric Syndrome. Archives of Gynecology \& Obstetrics 294(1):193-200. https://doi.org/ 10.1007/s00404-016-4078-x.

Eriksen Thomas Hylland

2017 What is Anthropology? London: Pluto Press.

Evans, Spencer C., Michael C. Roberts, Jared W. Keeley, Jennifer B. Blossom, Christina M. Amaro, Andrea M. Garcia, Cathleen Odar Stough, Kimberly S. Canter, Rebeca Robles, and Geoffrey Reed

2015 Vignette Methodologies for Studying Clinicians' Decision-Making: Validity, Utility, and Application in ICD-11 Field Studies. International Journal of Clinical and Health Psychology 15 (2):160-170. https://doi.org/10.1016/j.ijchp.2014.12.001.

Fu, Shu-fei, Chung-wah Cheng, Li Zhang, Linda Li-dan Zhong, Wai Kun, Jia Lin, Bo-li Zhang, Yong-yan Wang, Hong-cai Shang, and Zhao-xiang Bian

2016 Consensus-Based Recommendations for Case Report in Chinese Medicine (CARC). Chinese Journal of Integrative Medicine 22(1):73-79. https://doi.org/10.1007/s11655-015-2121-6.

Kirmayer, Laurence J., and Maxwell J. D. Ramstead

2017 Embodiment and Enactment in Cultural Psychiatry. In Embodiment, Enaction, and Culture: Investigating the Constitution of the Shared World. Christoph Durt, Thomas Fuchs, and Christian Tewes, eds., pp. 397-422. Cambridge (MA): MIT Press.

Kleinman, Arthur

1977 Depression, Somatization and the 'New Cross-Cultural Psychiatry'. Social Science \& Medicine 11(1):3-10.

1978 Concepts and a Model for the Comparison of Medical Systems as Cultural Systems. Social Science \& Medicine 12(2B):85-95.

1982 Neurasthenia and Depression: A Study of Somatization and Culture in China. Culture, Medicine and Psychiatry 6(2):117-190.

Krippendorff, Klaus

2004 Content Analysis: An Introduction to Its Methodology. Thousand Oaks (CA): Sage Publications.

Lee, Dominic T.S., Joan Kleinman, and Arthur Kleinman

2007 Rethinking Depression: An Ethnographic Study of the Experiences of Depression Among Chinese. Harvard Review of Psychiatry 15(1):1-8.

Mauss, Marcel

1985 A Category of the Human Mind: The Notion of Person: The Notion of Self. In The Category of the Person: Anthropology, Philosophy, History. Michael Carrithers, Steven Collins, and Steven Lukes, eds., pp. 1-25. Cambridge: Cambridge University Press.

McKee, M. Diane, Benjamin Kligler, Jason Fletcher, Francesca Biryukov, William Casalaina, Belinda Anderson, and Arthur Blank

2013 Outcomes of Acupuncture for Chronic Pain in Urban Primary Care. Journal of the American Board of Family Medicine 26(6):692-700. https://doi.org/10.3122/jabfm.2013.06.130003.

McQuade, Jennifer L., ZhiQiang Meng, Zhen Chen, Qi Wei, Ying Zhang, J. WenYing Bei, Lyn Palmer, and Lorenzo Cohen

2012 Utilization of and Attitudes Towards Traditional Chinese Medicine Therapies in a Chinese Cancer Hospital: A Survey of Patients and Physicians. Evidence-based Complementary and Alternative Medicine 2012:504507. https://doi.org/10.1155/2012/504507.

Miller, Greg

2006 China: Healing the Metaphorical Heart. Science 311(5760):462-463. https://doi.org/ 10.1126/science.311.5760.462.

Palinkas, Lawrence, Sarah Horwitz, Carla Green, Jennifer Wisdom, Naihua Duan, and Kimberly Hoagwood

2015 Purposeful Sampling for Qualitative Data Collection and Analysis in Mixed Method Implementation Research. Administration and Policy in Mental Health and Mental Health Services Research 42(5):533-544. https://doi.org/10.1007/s10488-013-0528-y.

Ryder, Andrew G., and Yulia E. Chentsova-Dutton

2012 Depression in Cultural Context: "Chinese Somatization," Revisited. Psychiatric Clinics of North America 35(1):15-36. https://doi.org/10.1016/j.psc.2011.11.006.

Ryder, Andrew G., Jiahong Sun, Xiongzhao Zhu, Shuqiao Yao, and Yulia E. Chentsova-Dutton

2012 Depression in China: Integrating Developmental Psychopathology and Cultural-Clinical Psychology. Journal of Clinical Child \& Adolescent Psychology 41(5):682-694. https://doi. org/10.1080/15374416.2012.710163. 
Suh, Soyoung

2013 Stories to Be Told: Korean Doctors Between Hwa-byung (Fire-Illness) and Depression, 1970-2011. Culture, Medicine and Psychiatry 37(1):81-104. https://doi.org/10.1007/s11013-012-9291-x.

Tao, Wei-Wei, Hua Jiang, Xiao-Mei Tao, Ping Jiang, Li-Yan Sha, and Xian-Ce Sun

2016 Effects of Acupuncture, Tuina, Tai Chi, Qigong, and Traditional Chinese Medicine FiveElement Music Therapy on Symptom Management and Quality of Life for Cancer Patients: A Meta-Analysis. Journal of Pain \& Symptom Management 51(4):728-747. https://doi.org/ 10.1016/j.jpainsymman.2015.11.027.

WHO Regional Office for the Western Pacific.

2007. WHO International Standard Terminologies on Traditional Medicine in the Western Pacific Region. WHO Regional Office for the Western Pacific. www.wpro.who.int/publications/who_ istrm_file.pdf

Wong, Wendy, L.K. Cindy Lam, Rita Li, Sze Hon Ho, Leung Kwok Fai, and Zhao Li

2011 A Comparison of the Effectiveness Between Western Medicine and Chinese Medicine Outpatient Consultations in Primary Care. Complementary Therapies in Medicine 19(5):264275. https://doi.org/10.1016/j.ctim.2011.07.001.

Yeung, Wing-Fai, Ka-Fai Chung, Ka-Yan Ng, Yu Yee-Man, Eric Tat-Chi Ziea, and Bacon Fung-Leung $\mathrm{Ng}$

2014 A Systematic Review on the Efficacy, Safety and Types of Chinese Herbal Medicine for Depression. Journal of Psychiatric Research 57:165-175. https://doi.org/10.2016/j. jpsychires.2014.05.016.

Yeung, Wing-Fai, Ka-Fai Chung, Ka-Yan Ng, Yu Yee-Man, Shi-Ping Zhang, Bacon Fung-Leung Ng, and Eric Tat-Chi Ziea

2015 Prescription of Chinese Herbal Medicine in Pattern-Based Traditional Chinese Medicine Treatment for Depression: A Systematic Review. Evidence-Based Complementary \& Alternative Medicine 2015:160189. https://doi.org/10.1155/2015/160189.

Zhou, Xue-sheng, ed.

2006 中醫基礎理論圖表解 [Zhong Yi Ji Chu Li Lun Biao Tu Jie, Basic Theories of TCM Explained in Diagrams and Tables]. Tainan: Tafu Book.

Publisher's Note Springer Nature remains neutral with regard to jurisdictional claims in published maps and institutional affiliations. 